\title{
MAKING REPUTATION SYSTEM TRACEABLE WITHOUT LOSING PRIVACY
}

\author{
Keli Zhang, Yi Xian Yang
}

Preliminary note

In existing reputation system nodes usually adopt regular pseudonyms instead of true identities to gain the anonymity. However complete anonymity will cause watershed and Sybil attack, which look on system be out of control and break the fairness of the reputation system. This paper introduces the conditional anonymity mechanism to check the evaluation between anonymity nodes, evaluation between two nodes if not more than the several times, but not be evaluated between two pseudonyms belong to the same peer. It will be effective, otherwise, the node true identity will be exposed, if number of attacks of the peer within the time exceeds $d$ times, nodes in the evaluation and transaction will be tracked. Analysis shows that this mechanism cannot only protect the identity of peer's anonymity, but identify and track malicious attackers.

Keywords: conditional anonymity; privacy; pseudonym; reputation system; traceable

\section{Izrada sljedivog sustava ugleda bez gubitka privatnost}

Prethodno propćenje U postojećim čvorovima sustava ugleda obično se prihvaćaju redoviti pseudonimi umjesto pravog identiteta radi postizanja anonimnosti. Međutim, potpuna anonimnost će uzrokovati prekretnicu i Sybil napad, koji bi sustav ugleda stavio izvan kontrole i razbio pravičnost sustava ugleda. Ovaj rad predstavlja uvjetni mehanizam anonimnosti za provjeru vrednovanja između anonimnosti čvorova, vrednovanja između dva čvora ako se ne ponavlja nekoliko puta, ne može se vrednovati između dva pseudonima koji pripadaju istoj razini. On će biti učinkovit, ako će pravi identitet čvora biti izložen, ako je broj napada iste razine unutar vremena koje prelazi $d$ vremena, čvorovi u procjeni i transakciji će biti praćeni. Analiza pokazuje da ovaj mehanizam ne samo da može zaštititi identitet istorazinske anonimnosti, već će identificirati i pratiti zlonamjerne napadače.

Ključne riječi: privatnost; pseudonim; sljediv; sustav ugleda; uvjetna anonimnost

\section{Introduction}

The basic idea of trust and reputation model is to allow mutual evaluation between both sides of a deal [1]. The evaluation will help other nodes determine whether to make a deal with the node being evaluated. The reputation of a user depends entirely on its identity. In order to accumulate its reputation value, a user will normally use its real identity or use a pseudonym for a long time. But a long-term use of the same identity will link all the exchange information of a user together. Malicious users or outlaws will systematically analyse the data. All the activities and hobbies of a user will be exposed. This will pose a grave threat to the privacy of users [2].

In order to ensure complete anonymity, users need to change their pseudonyms periodically, which will incur the conflict between privacy and reputation, render reputation evaluation uncontrollable, and reduce the accountability of the reputation system. Truthful users may suffer from malicious users while malicious users can go unpunished. It is because malicious users abandon their previous pseudonyms along with the reputation values, and regain a new one with an initial value. Besides, due to lack of controllability, two users can collude with each other and make false mutual evaluations to increase their reputation value; malicious users will generate many pseudonyms to evaluate themselves, increasing their reputation value.

To solve the above-mentioned problems, this paper introduces evaluative mechanism, conditional anonymity in reputation system. Each user will first acquire an evaluation container from TTP based on CL protocol, and generate a Rater certificate and a Ratee certificate. When the user submits its reputation value to TTP, Rater certificate and Ratee certificate will be submitted as well. Evaluation certificates will anonymously monitor reputation evaluations between nodes. Only if the following anonymity conditions are met: 1) no ramming attack or aspersion; 2) no Sybil attack; can a node gain the reputation value, otherwise, the real identity of a node will be exposed. If a node is attacked d times or more in a limited amount of time, all evaluation and exchange information of the node will be traced. In this protocol, reputation value is bound to the fixed identity (public key or other permanent identity) to strike a balance between anonymity and reputation and ensure the privacy of identity when the reputation value is updated based on blind signature scheme.

\section{Related work}

At present, among researches on reputation system, most focus on building distributed reputation systems

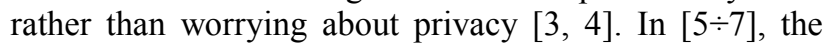
works only address the conflict issue of anonymity in reputation system. In $[8,9]$, users use vague identities, but all their activities will still be linked. Authors [2, 10] allow a user to have multiple pseudonyms, but transaction information of each pseudonym will be linked by attackers. In $[11,12]$, peers gain anonymity through concealing their pseudonyms. But these systems still cannot ensure completely anonymity of users. In $[13,17]$, users periodically change their pseudonyms to ensure the anonymity of their identity and cut the links between pseudonyms. But in [13], the author only ensures that the e-cash as the reputation value cannot be repeatedly used, but does not control the frequency of evaluation for the two pseudonyms. In [17], the author introduces two TTPs to control the frequency of evaluations between two users, but the real identity of the two users is exposed to a thirdparty, secure multiparty computation [14] and Homomorphic encryption [16] is proposed to protect the identity of rater, but the protocol cannot to identity and 
resist the Malicious attacks. Recently a new cryptographic primitive called signature of reputation was proposed in [15].

Conditional anonymity remains an important research subject. At present, research is mainly focused on e-cash $[18,19]$. Another kind of research is focused on key escrow [20, 21]. Recent correlation study [22, 23] is about a user being able to only use $k$ anonymous and unlinked identities in a limited amount of time. Conditional anonymity in reputation system is rarely touched upon in research.

\section{Preliminaries}

Preliminaries are classified into bilinear map, complexity assumptions and cryptographic tool. The main classification is as follows:

\subsection{Bilinear map}

Assume $G_{1}, G_{2}, G_{T}$ are cyclic groups with the exponent being prime number $\mathrm{q}$, and $g_{1}$ is the generator of $G_{1}, g_{2}$ the generator of $G_{2} ; \eta$ is the isomorphism that can realize the calculation from $G_{2}$ to $G_{1}$, $\eta\left(g_{2}\right)=g_{1} ; e$ is a bilinear map: $e: G_{1} \times G_{2} \rightarrow G_{T}$. If the following condition is met, then the group $\left(G_{1}, G_{2}\right)$ is a bilinear group: 1) bilinearities: $g_{1} \in G_{1}, g_{2} \in G_{2}$, $a, b \in Z_{q}, e\left(g_{1}{ }^{a}, g_{2}{ }^{b}\right)=e\left(g_{1}, g_{2}\right)^{a b}$ are given; 2) nondegeneracy: $\left.e\left(g_{1}, g_{2}\right) \neq 1 ; 3\right)$ computability: map $e$ can effectively compute any input pairs.

\subsection{Complexity assumptions}

\section{- Strong RSA assumption}

Strong RSA assumption [24]: Assuming $n=p q$ is the RSA modulus, $\left(p=2 p^{\prime}+1, q=2 q^{\prime}+1\right.$ and $p, p^{\prime}$, $q^{\prime}$ are prime numbers) component $g \in Z_{n}{ }^{*}$, when $e>1$ and the condition $h^{e} \equiv g \bmod n$ is met, it is difficult to find a two-tuples $(h, e)$.

\subsection{Cryptographic tool}

\section{- CL signature}

Camenish-Lysyanskaya (CL signature) [25] scheme is based on strong RSA assumption. It is between a user and a signer, allowing the user to acquire a signature $\sigma$ about the question $\mathrm{C}$ ( $\mathrm{C}$ is a specific question about $\left.\left(v_{1}, \ldots, v_{l}\right)\right)$. But the signer does not know the exact value of $\mathrm{C}$. The signer calculates $C \operatorname{LSign}(C)$ and sends it to the user, who will get $\sigma=\operatorname{sign}\left(v_{1}, \ldots, v_{l}\right)$, and be able to verify the signature through $\operatorname{Verif}\left(\sigma,\left(v_{1}, \ldots, v_{l}\right)\right)=1$. When the user verifies the signature, besides giving necessary information to the signer, he also has to send to the signer the signature of knowledge that holds information on the value of $\mathrm{C}\left(v_{1}, \ldots, v_{l}\right)$.

\section{- DY Verifiable Random Function}

Assume $G=\langle g\rangle$ is a group with the exponent being q (a large prime number), $s \in Z_{q}$. This model adopts verifiable random function $F_{g, s}{ }^{D Y}(x)=g^{1 /(x+s+1)}$ $x \in Z_{q}^{*}$ put forward by Dodia and Yampolskiy [26]. This function is secure under Y-DDHI.

- Pedersen commitment

- Pedersen [27] puts forward a commitment scheme on discrete logarithm problem. The public parameter is the prime exponent $\mathrm{q}$ of the group. The generated components $\left(g_{0}, \ldots, g_{m}\right)$, and commitment components $\left(v_{1}, \ldots, v_{m}\right) \in Z_{q}{ }^{m}$. Choose a random number $r \in Z_{q}$,

- $\quad C=\operatorname{Ped} \operatorname{Com}\left(v_{1}, \ldots, \mathrm{v}_{\mathrm{m}} ; \mathrm{r}\right)=\mathrm{g}_{0}{ }^{\mathrm{r}} \coprod_{i=1}^{m} g_{i}^{v^{i}}$.

\section{- Blind signature}

D. Chuam first put forward the term blind signature [28]. Blind signature means that someone needs to sign for some data, unaware of the contents of the data. Compared with normal digital signatures, blind digital signature has two prominent features: (1) the contents of the information are not known to the signer; (2) after the signature is publicized by the recipient, the signer cannot trace the signature.

\section{- Known discrete-logarithm-based, zero-knowledge proofs}

This paper uses the identification of zero-knowledge proof based on discrete logarithm put forward by Camenisch and Stadler [30]. For example: $P K\left\{(\partial, \beta, \delta): y=g^{\partial} h^{\beta} \wedge \tilde{y}=\widetilde{g}^{\partial} \widetilde{h}^{\beta}\right\}$, denotes the zeroknowledge proof of $\partial, \beta, \delta$, and $y=g^{\partial} h^{\beta}, \tilde{y}=\widetilde{g}^{\partial} \tilde{h}^{\beta}$ are tenable, where $y, g, h, \tilde{y}, \widetilde{g}, \tilde{h}$ are components of $G=<g>=<h>$ and $\widetilde{G}=<\widetilde{g}>=<\tilde{h}>$. In the meantime, Fait-Shamir [29] heuristic can be used to turn zeroknowledge proof into knowledge signature of $m$, written as: $\operatorname{SPK}\left\{(\alpha): y=g^{\partial}\right\}(m)$.

\section{General descriptions}

This chapter will introduce assumptions of the system, participants and general designing ideas, etc.

\subsection{Assumptions}

Network environment: Assume the whole reputation system is situated in anonymous network (an Onion Router, Mixnet, etc.).

Pseudonym: Many pseudonyms will be deposited at every node in advance. The validity of pseudonyms will be verified by TTP.

\subsection{Participating entities}

Node(User): A node is a normal node in the network. Each node plays two different roles: as a Rater, it gives 
The evaluation values to other nodes, or as a Ratee, it receives others' evaluation of itself. Transaction and evaluation between two nodes is realized through pseudonym, which is also used to show the reputation value to other users.

TTP (Trusted Third Party): TTP controls information related to the reputation of each node. It creates a reputation account for each user based on the user's public key. It is assumed that TTP is trusted when verifying pseudonyms and calculating the reputation value, but not trusted when it comes to user's privacy.

\subsection{General designing ideas}

Assume the CL signature key pair of TTP and user's key pair are $\left(p k_{T T P}, s k_{T T P}\right)$ and $\left(p k_{u}, s k_{u}\right)$ respectively, where $p k_{u}=g^{s k_{u}} . F_{g, s}{ }^{D Y}$ is the DY pseudo-random function. Each user will first anonymously acquire a certificate container from TTP. The container consists of the key seed s and a user's key $s k_{u}$ of $F_{g, s}{ }^{D Y}$, and the CL signature of TTP $\left(s k_{u}, s\right)$. CL signature ensures that TTP will not know s and $s k_{u}$. After a deal is over, Rater first generates evaluation certificate Cert $_{\text {rater }}$ and submits it to Ratee. Cert $t_{\text {rater }}$ consists of: tag of the certificate $S=F_{g, s}{ }^{D Y}\left(R_{1}\right) \quad, \quad$ double evaluation equation $T=p k_{u} F_{g, s}{ }^{D Y}\left(R_{2}\right)^{r}$ and ZKPOK $\phi$ (which proves that $\mathrm{S}$ and $\mathrm{T}$ are validly generated by $\left.\sigma_{T T P}\left(s k_{u}, s\right)\right) . R_{1}$ and $R_{2}$ are related to Ratee's pseudonym. Subsequently, Ratee generates Ratee certificate Cert $_{\text {ratee }}$, Ratee certificate is similar to evaluative certificate, consisting of the tag of certificate $V=F_{g, s_{1}}{ }^{D Y}\left(R_{1}\right)$, double evaluation equation $W=p k_{u} F_{g, s_{1}}{ }^{D Y}\left(R_{2}\right)^{t}$ and ZKPOK $\varphi . R_{1}$ and $R_{2}$ are the same with $R_{1}$ and $R_{2}$ in the evaluation certificate. Ratee submits Cert $_{\text {rater }}$ and Cert $_{\text {ratee }}$ to TTP. TTP verifies Cert $_{\text {rater }}$ and Cert $t_{\text {ratee }}$. If they are valid, TTP temporarily deposits Cert $_{\text {rater }}$ and Cert $_{\text {ratee }}$. If they are invalid, TTP abandons Cert rater $_{\text {and }}$ Cert $t_{\text {ratee }}$.

If Rater evaluates the same user twice or more in a limited amount of time, two evaluation certificates Cert $_{\text {rater }}$ must use the same evaluation tag S, and the two Ratee certificates Cert $_{\text {ratee }}$ must use the same tag V as well. Thus, TTP can acquire the public key of Rater and Ratee based on double evaluation equation: $p k_{\text {rater }}=\left(\frac{T_{2}^{r_{1}}}{T_{1}^{r_{2}}}\right)^{\left(r_{1}-r_{2}\right)^{-1}}$ and $p k_{\text {ratee }}=\left(\frac{W_{2}^{t_{1}}}{W_{1}^{t_{2}}}\right)^{\left(t_{1}-t_{2}\right)^{-1}}$; if both pseudonyms of the same user make evaluations, evaluative certificate tag $\mathrm{V}$ and Ratee certificate tag $\mathrm{S}$ will be the same. TTP can acquire the public key of the violator $p k=\left(\frac{T^{t}}{W^{r}}\right)^{(t-r)^{-1}}$ based on double evaluation equation $\mathrm{T}$ and $\mathrm{W}$. TTP records the identity of the malicious violator that gets caught. When the node updates its reputation value to TTP with the same public key, it must submit a sub-key $s_{i}$ of the evaluation container. $s_{i}$ is generated through verifiable key sharing mechanism. Each $s_{i}$ contains a verifiable secret piece. Once the number of $s_{i}$ acquired from a certain node by TTP exceeds the threshold, the s of the node's evaluation container will be leaked, and all the node's evaluations will be traced.

\section{Security model}

\subsection{The definition of general security}

The protocol is mainly composed of the following protocols and algorithms:

- TTPKeygen $\left(1^{k}\right.$, params) : The key-generation algorithm of TTP. Input the system's public parameter $1^{k}$ and the identity of TTP, the output will be the public/private key pair of TTP $\left(p k_{T T P}, s k_{T T P}\right)$.

- UserKeyGen $\left(1^{k}\right.$,params $)$ : The key-generation algorithm of the node. Input the system's parameter $1^{k}$ and the identity of the node ID, the output will be the public/private key pair of the node $\left(p k_{u}, s k_{u}\right)$.

- Withdraw $\left(U\left(p k_{u}, s k_{u}\right), T T P\left(p k_{T T P}, s k_{T T P}\right)\right)$ Evaluation certificate withdrawal protocol. The node acquires evaluation certificate container from TTP. The node's output will be evaluation container $\mathrm{W}$, or false information.

- $\quad \operatorname{RaterCert}\left(U\left(W, p k_{M}\right), M\left(s k_{M}, p k_{T T P}, P_{M}\right)\right)$

Evaluation certificate-generation protocol. Node U (using pseudonym $P_{U}$ ), based on the evaluation certificate container, generates evaluative certificate Cert $_{\text {rater }}$ on node $\mathrm{M}$ (using pseudonym $P_{M}$ ). M acquires the evaluation tag $\mathrm{S}$ of Cert $_{\text {rater }}$ and the validity proof of the identification $\pi$.

- $\quad$ RateeCert $\left(M\left(W, p k_{T T P}\right), T T P\left(s k_{T T P}, p k_{T T P}, P_{M}\right)\right)$ Evaluated certificate-generation protocol. Node $M$ (using pseudonym $P_{M}$ ) submits Ratee certificate Cert $_{\text {ratee }}$ to TTP. TTP acquires the evaluated identification $\mathrm{V}$ of $\mathrm{M}$ and the validity proof of the identification $\varphi$.

- $\quad \operatorname{VerifyRep}\left(\mathrm{M}\left((S, \pi),(V, \varphi), p k_{T T P}\right), T T P\left(p k_{T T P}, s k_{T T P}\right)\right)$ Reputation evaluation verifying protocol. If Rater and Ratee are both trusted, TTP will accept the Ratee certificate and Rater certificate submitted by Ratee M (using pseudonym $P_{M}$ ).

- $\quad \operatorname{RepDeposit}\left(\mathrm{M}\left(\sigma, p k_{T T P}\right), T T P\left(p k_{T T P}, s k_{T T P}\right)\right)$ Reputation value depositing protocol. If the blind signature is valid, TTP will deposit and update the new reputation value of node $\mathrm{M}$.

- Identity(params, $S, \pi_{1}, \pi_{2}$ ) Attacker identification algorithm. The system can identify the same certificate tag $\mathrm{S}$ of the attacker, and the two identification proofs $\pi_{1}$ and $\pi_{2}$. The output of the algorithm is a public key $p k_{u}$ and the proof $\Pi_{G}$.

- VerifyGuilt(params, $S, \Pi_{G}, p k_{u}$ ) Guilt verifying algorithm. It allows publicizing the guilt proof $\Pi_{G}$. 


\subsubsection{Soundness}

Rater can evaluate its deal-maker through the evaluation certificate container. But the frequency of evaluation cannot exceed a limit. A knowledge extractor $\mathrm{K}$ is given. $\mathrm{K}$ and the adversary execute the certificate withdrawal protocol Withdraw, extracting m evaluation certificate tags $S_{1}, S_{2} \ldots . S_{m}$. For each evaluation certificate identification of the adversary, when $S \neq S_{i} \forall 1 \leq i \leq m$, the probability that TTP accepts $(S, \pi)$ as a valid evaluation certificate is negligible.

\subsubsection{Identification of the attacker}

Assume TTP is truthful, and $U_{1}$ and $U_{2}$ are both truthful users. When $U_{1}, U_{2}$ and the adversary execute the evaluation certificate-generation protocol, the output of $U_{1}$ will be $\left(S, \pi_{1}\right)$, the output of $U_{2}\left(S, \pi_{2}\right)$. This guarantees high probability that the public key $p k_{u}$ and proof $\Pi_{G}$ generated from Identity (params, $\left.S, \pi_{1}, \pi_{2}\right)$ are accepted by VerifyGuilt(params, $S, \Pi_{G}, p k_{u}$ ) .

\subsubsection{Anonymity}

TTP cannot acquire any information on Ratee or Rater even if it colludes with malicious users. This protocol introduces simulator $\mathrm{S}$. $\mathrm{S}$ has some information that other participants cannot know. For example: in a normal parameter model, $\mathrm{S}$ itself generates information on parameters. In a random oracle model, $\mathrm{S}$ is the controlled random oracle. Simulator $\mathrm{S}$ should be able to simulate evaluation certificates (Rater certificate and Ratee certificate) without accessing the certificate container. The simulated certificate cannot be distinguished from the valid certificate. To be more specific, $\mathrm{S}$ can execute certificate-generation protocol (Rater certificate and Ratee certificate) without knowing secret $\mathrm{s}$ of the certificate container or accessing the container.

\subsubsection{Excludability}

Assume the adversary participated in the execution of the certificate-withdrawal protocol of a truthful node with the public key being $p k_{u}$, and subsequently in the execution of certificate-generation protocol of the user. When an attacker inputs a public key certificate $p k_{u}$ and a forged proof $\Pi_{G}$, and claims the user is the attacker, the probability that forged proof $\Pi_{G}$ gets accepted by VerifyGuilt(params, $S, \Pi_{G}, p k_{u}$ ) is negligible.

\subsection{Definition of formal security}

The definition of formal security depends on knowledge extractor $\mathrm{K}$ and the simulator $\mathrm{S}$. For a given deal, the knowledge extractor $\mathrm{K}$ and extract the evaluated certificate tag of the adversary (when unaware of the node's key or the secret of the certificate container, simulator S) can simulate the evaluation certificategeneration protocol and the evaluated certificategeneration protocol. Under normal circumstances, the definition of knowledge extractor $\mathrm{K}$ and the simulator $\mathrm{S}$ depends on provable security model. To make the definition more general, a special protocol like a knowledge proof or zero-knowledge proof is needed. For example: a knowledge proof in the withdrawal protocol Withdraw, while a zero-knowledge proof in the evaluation certificate (Ratee certificate) generation protocol.

$X-Y$ is used to represent the security model, $X \in(I O, B B, N B B)$, (where IO represents the inputoutput model, BB represents black-box model, and NBB represents non-black-box model), $Y \in(K, R O)$. The algorithm (Alg) with which Adversary A accesses $X-Y$ model is $A \lg ^{X-Y(A)}$. In some models of public-parameter, knowledge extractor $\mathrm{K}$ and simulator $\mathrm{S}$ are allowed to access some auxiliary information (the auxiliary information will not be provided to participants. The information knowledge extractor $\mathrm{K}$ and simulator $\mathrm{S}$ access respectively are represented by auxext and auxsim.

In the $X-Y$ model in the language $\mathrm{L}$, $\mathrm{K}_{\text {Prot, } L}^{X-Y(A)}$ (params,auxext, $\left.x\right)$ represents the knowledge extractor of knowledge proof protocol (Prot), while $S_{\text {Prot }, L}^{X-Y(A)}$ (params, auxsim, $x$ ) represents zero-knowledge simulator of proof protocol (Prot).

\subsubsection{Validity}

To make it easier to analyse, Withdraw protocol is divided into three parts: Withdraw $w_{b}$, Withdraw $_{m}$ and Withdraw $_{e}$, respectively denoting the beginning, the middle and the ending of the protocol (letters b, m, e represents beginning, middle and ending). Withdraw ends when the node sends message to TTP. Withdraw ends when TTP sends message to the user. Withdraw $w_{e}$ is taken as the proof protocol. The user is the prover, TTP verifier. Withdraw $w_{m}$ output from the verifier determines whether TTP will continue Withdraw $w_{e}$, and send the ending message to the peer. $m_{1}$ represents the first information the peer will send to TTP. $b_{1}$ represents the state information when TTP received $m_{1}$. The detailed requirements of validity are as follows:

a) Whatever model is given, there exists a highly efficient interpretable language $L_{S}$ and a knowledge extractor $\mathrm{K}_{\mathrm{Prot}, L}^{X-Y(A)}\left(\right.$ params,auxext, $\left.b_{1}, m_{1}\right)$. All $b_{1}$ are calculated by TTP. All $m_{1}$ are extracted tags $\alpha=\left(S_{1}, S_{2} \ldots . . S_{m}\right.$, aux $),\left(b_{1}, m_{1}, \alpha\right) \in L_{S}$. In Withdraw ${ }_{m}$ the probability that TTP accepts $m_{1}$ is not negligible.

b) With regard to input ( arams, $p k_{T T P}$ ), adversary $A$ executes the game as follows: $A$ plays withdrawal protocol with TTP and reputation verifying protocol VerifyRep for any times. If the ist withdrawal protocol Withdraw succeeds, then the output of knowledge 
extractor $\mathrm{K}_{\text {Prot,L }}^{X-Y(A)}$ (params,auxext, pk $\left.k_{i}, b_{1, i}, m_{1, i}\right)$ will be $\left(b_{1, i}, m_{1, i}, \alpha_{i}\right) \in L_{S}$; the evaluation identifications list acquired by $\mathrm{f}$ executing Withdraw is represented by $A_{f}=\left(S_{i, j} \mid \leq i \leq f, 1 \leq j \leq m\right)$. For some $\mathrm{f}$, in reputation verifying protocol, if the honest TTP accepts evaluation identification $S \notin A_{f}, A$ wins. It needs to be proven that the probability is negligible that adversary A wins the game within probabilistic polynomial time (PPT).

\subsubsection{The identification of attacker}

The feature of identifying the attacker ensures that within PPT time, the probability that the adversary $A$ wins the following game is negligible. Public parameter model assumes params and auxext are fixed.

With regard to input (params, $p k_{T T P}$ ), adversary $A$ executes the game as follows: A executes Withdraw and reputation verifying protocol VerifyRep with TTP. If the withdrawal protocol is executed successfully, the output of the knowledge extractor $\mathrm{K}_{\text {Prot,L }}{ }^{X-Y(A)}$ will be $\left(b_{1, i}, m_{1, i}, \alpha_{i}\right) \in L_{S} ; A_{i}$ refers to the evaluation tag of the node with the public key being $p k_{i}$. In the reputation verifying protocol VerifyRep, if TTP accepts twice the same evaluation identification $S \in A_{i}$, the attacker identifying algorithm Identity(Params, $\left.S, \pi_{1}, \pi_{2}\right)$ generates $p k_{i}$ and the proof $\Pi_{G}$. The probability is relatively high that VerifyGuilt(params, $S, \Pi_{G}, p k_{u}$ ) accepts the public key $p k_{i}$ and the proof $\Pi_{G}$.

\subsubsection{Anonymity}

Assume adversary $A$ inquires whatever information with the public key $p k_{T T P}$ :

a) Public key of the inquiry node $\mathrm{j}$ : $A$ requests and accepts the public key $p k_{j}$ of $\mathrm{j}$, and generates valid and truthful key pair $\left(p k_{j}, s k_{j}\right)$.

b) Withdraw of node j: $A$ and $\mathrm{j}$ execute Withdraw $\left(U\left(p k_{u}, s k_{u}\right), A(\right.$ state $\left.)\right)$, state refers to the state of $A$. The ist Withdraw inquiry is represented by $W_{i}$. If $W_{i}$ is false information, $W_{i}$ is the invalid certificate container.

c) RaterCert of j: $A$ executes RaterCert generation protocol based on the container W: RaterCert $\left(U\left(W_{j}\right), A(\right.$ state $\left.)\right)$.

If $\mathrm{A}$ only makes reputation evaluation based on valid certificate container, but never makes evaluations of violations, then $A$ is valid.

An existing simulator $S^{X-Y(A)(.)}$ (params, auxsim,.) is needed. No valid adversary $A$ can distinguish whether it is playing Game R(Real) or Game I(Ideal):

Game R: The answer to the question A inquires is shown above.
Game I: The public key A inquires and Withdraw answer are shown above. With regard to reputation evaluation certificate generation protocol RaterCert, A interacts with simulator $S^{X-Y(A)(.)}$ (params, auxsim, $p k_{T T P}$ ) $\operatorname{not} U\left(W_{j}\right)$.

\subsubsection{Excludability}

Excludability ensures that only nodes that make malicious attacks need to be punished, while honest nodes need not. There are two kinds of excludability: weak excludability and strong excludability. Weak excludability punishes nodes that make attacks, while strong excludability not only punishes nodes that make attacks, but also makes malicious attackers accountable for their behaviour. This paper considers only weak excludability. To define the weak excludability in this chapter, assume adversary $A$ will make the following attacks on user U:

a) Initialization Adversary $A$ acquires the public key of TTP through attacks.

b) Inquiry Adversary $A$ inquires node $U$ as such:

Withdraw: The adversary fakes its identity as TTP and executes certificate withdrawal protocol Withdraw with the user. The user outputs the evaluation certificate container $W$.

RaterCert: The adversary fakes its identity as Ratee, and executes evaluation certificate generation protocol with Rater. Rater's output is the evaluation certificate container $W$.

Success criterion: When the protocol ends, if VerifyGuilt(params, $S, \Pi_{G}, p k_{u}$ ) accepts adversary A's output $\left(S, \Pi_{G}\right)$, then $A$ wins the game.

\section{Detailed description of the protocols}

This chapter introduces the initialization, evaluation certificate withdrawal protocol (Withdraw), evaluation Rater certificate-generation protocol (RaterCert), Ratee certificate-generation protocol (RateeCert), reputation evaluation verifying protocol (VerifyRep), reputation value updating protocol (RepDepost), attacker identifying algorithm (Identity) and tracing malicious nodes.

\subsection{Initialization}

\section{- Instructions on the parameter}

$G_{1}=<g_{1}>, \mathrm{n}$ is the modulus of special RSA. $g_{1}$ is the quadratic residue modulus $\mathrm{n}, h_{1} \in G_{1}$. Pedersen commitment and CL signature based on RSA both use this parameter.

$$
G_{2}=<g_{2}>, g_{2} \text { is the prime exponent } q=\Theta\left(2^{k}\right),
$$
$h_{2} \in G_{2}$. Certificate identification, double evaluation equation both use this parameter and $F_{g, s}{ }^{D Y}(x)=g_{2}{ }^{1 /(x+s+1)}$.

\section{- Key generation}

TTP key generation: by executing TTP key generation algorithm TTPKeygen $\left(1^{k}\right.$, params $)$ g CL signature key pair and blind signature key pair of TTP 
$\left(p k_{T T P}, s k_{T T P}\right)$ and $\left(p k_{T T P}{ }^{\sigma}, s k_{T T P}{ }^{\sigma}\right)$ respectively will be generated;

User's key generation: by executing user's key generation algorithm UserKeyGen $\left(1^{k}\right.$, params) , and inputting the system parameter $1^{k}$ node's identity ID, user public/private key pair $\left(p k_{u}, s k_{u}\right)$ will be generated.

\subsection{Evaluation certificate container withdrawal protocol}

Assume the user and TTP authenticate each other. The protocol with which the user acquires evaluation certificate container from TTP is as follows. The protocol is executed based on CHL e-cash withdrawal protocol:

1) The user uses the real identity to negotiate a commitment value $C^{\prime}$ with TTP with regard to $s \in Z_{q}$ : the user chooses a random number $s^{\prime} \in Z_{n}^{*}$, and calculates Pedersen commitment $C^{\prime}=\operatorname{Ped} \operatorname{Com}\left(s k_{u}, s^{\prime} ; r\right)=g_{0}^{r} g_{1}^{s k_{u}} g_{2}^{s^{\prime}}(\operatorname{modn})$. The user sends $C^{\prime}$ and $p k_{u}$ to TTP, as well as the corresponding secret $\mathrm{s}$ to $C^{\prime}$ and the zero-knowledge proof of the user's private key $s k_{u}$.

2) TTP first verifies the zero-knowledge signature $\phi$. If it is correct, TTP chooses a random number $r^{\prime} \in Z_{n}^{*}$, calculates $C=C^{\prime} g_{0}{ }^{r^{\prime}}(\operatorname{modn})$, and executes $\mathrm{CL}$ signature $\sigma_{T T P}=C^{1 / e} \bmod n=\left(g_{0}{ }^{s^{\prime}} g_{1}{ }^{s k_{u}} g_{2}{ }^{r} g_{0}{ }^{r^{\prime}}\right)^{1 / \mathrm{e}}$. TTP sends $\sigma_{T T P}$ and $r^{\prime}$ to the user, and deposits the acquired $\mathrm{C}, p k_{u}, \phi, r^{\prime}, \sigma_{\text {TTP }}$ into the database, used to identify malicious users.

3) The user calculates $s=\left(s^{\prime}+r^{\prime}\right) \bmod n$, and verifies the CL signature of TTP, or verifies whether the following equation is tenable:

$$
\sigma_{T T P}^{e}=\left(g_{0}{ }^{s} g_{1}{ }^{s k_{u}} g_{2}^{r}\right)
$$

If it is, the user can confirm it is the signature of TTP.

4) The user certificate container is $W=\left(s k_{u}, s, \sigma_{T T P}\left(s k_{u}, s\right)\right)$, where $\mathrm{s}$ is the secret of the evaluation certificate container, $\sigma_{T T P}\left(s k_{u}, s\right)$ is the signature of TTP. According to $\mathrm{CL}$ signature protocol, TTP cannot know the values of $s k_{u}, s$.

\subsection{Evaluation certificates generation protocol}

Two users $\mathrm{U}$ and $\mathrm{M}$ use $P_{U}$ and $P_{M}$ to make a deal. After the deal, $P_{U}$ evaluates $P_{M} \cdot P_{U}$ will generate evaluation certificate Cert $_{\text {rater }}$ based on the information on $P_{M}$ 's pseudonym and its own certificate container. The process of $P_{U}$ generating Cert $_{\text {rater }}$ is as follows:

1) $P_{M}$ calculates $R_{1}=H\left(0 \Pi P_{M}\right), R_{2}=H\left(1 \Pi P_{M}\right) ; H($.$) is a$ one-way hash function with strong collision resistance;

2) $P_{M}$ sends the random number $r$ to $P_{U}$, where $r \in Z_{q}$;

3) $P_{U}$ sends the certificate identification and double evaluation Eq. $S=F_{g, s}{ }^{D Y}\left(R_{1}\right) ; T=p k_{u} F_{g, s}{ }^{D Y}\left(R_{2}\right)^{r}$

4) $P_{U}$ sends ZKPOK $\phi$ of $\left(R_{1}, R_{2}, s k_{u}, s, \sigma_{T T P}\left(s k_{u}, s\right)\right)$ to $P_{M}$ :

- $\quad R_{1}=H\left(0 \Pi P_{M}\right)$;

- $\quad R_{2}=H\left(1 \Pi P_{M}\right)$;

- $S=F_{g, s}{ }^{D Y}\left(R_{1}\right)$;

- $T=p k_{u} F_{g, s}{ }^{D Y}\left(R_{2}\right)^{r}$;

- $\operatorname{VerifySig}\left(p k_{T T P},(s k u, s), \sigma_{T T P}\left(s k_{u}, s\right)\right)=$ true ZKPOK in 4) is acquired in the following way:

a) $P_{U}$ calculates $B=\operatorname{Ped} \operatorname{Com}\left(s k_{u}\right), C=\operatorname{Ped} \operatorname{Com}(s)$; and proves the commitment values of $\mathrm{B}$ and $\mathrm{C}$ are $\mathrm{CL}$ signatures of TTP;

b) It should be proven that $S=F_{g, s}{ }^{D Y}\left(R_{1}\right)=g_{2}{ }^{1 / s+R_{1}+1}$ and $T=p k_{u} F_{g, s}{ }^{D Y}\left(R_{2}\right)^{r}=g_{2}{ }^{s k_{u}+r /\left(R_{2}+s+1\right)}$, and the result of knowledge signature is $\phi$.

5) $P_{M}$ verifies the validity of $\operatorname{Cert}_{\text {rater }}=(S, \pi=(r, T, \phi))$. If it is valid, $P_{M}$ temporarily saves the rater certificate Cert $_{\text {rater }}$.

\subsection{Evaluated certificate generation protocol}

$P_{M}$ generates evaluated certificate Cert $_{\text {ratee }}$ based on the information on its own pseudonym and its certificate container W. Cert $_{\text {ratee }}$ is generated in the same way as Cert $_{\text {rater }}, \operatorname{Cert}_{\text {ratee }}=(V, \psi=(W, t, \omega))$ where:

$V=F_{g, s_{1}}{ }^{D Y}\left(R_{1}\right)=g_{2}^{1 / s_{1}+R_{1}+1}$

$W=p k_{u} F_{g, s_{1}}{ }^{D Y}\left(R_{2}\right)^{t}=g_{2}{ }^{s k_{u}+t /\left(s_{1}+R_{2}+1\right)}, R_{1}=H\left(0 \Pi P_{M}\right), R_{2}$

$=H\left(1 \Pi P_{M}\right) \cdot s_{1}$ is the secret of evaluation certificate container of. $\mathrm{t}$ is randomly generated by $P_{M} . \omega$ is the noninteractive zero-knowledge signature.

\subsection{Reputation evaluation verifying protocol}

1) $P_{M}$ submits Rater certificate Cert $_{\text {rater }}$ and Ratee certificate Cert $_{\text {ratee }}$ to TTP. TTP first verifies $\phi$. If $\phi$ is not valid, TTP abandons $\operatorname{Cert}_{\text {rater }}=(S, \pi=(r, T, \phi))$; if $\phi$ is valid, TTP temporarily saves Cert $_{\text {rater }}$ submitted by $P_{M}$.

2) TTP subsequently verifies $\omega$. If $\omega$ is not valid, TTP

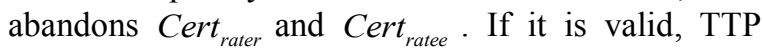
needs to further verify in the following way: 1) whether S equals $\mathrm{V} ; 2$ ) whether $\mathrm{S}$ is the same with all the $\mathrm{S}$ in Cert $_{\text {rater }}$ being deposited. If the above two conditions are met, TTP accepts Cert $_{\text {rater }}$ and Cert ${ }_{\text {ratee }}$, sends feedback message of reputation evaluation verified to $P_{M}$, and deposits $\operatorname{Cert}_{\text {rater }}$ and $\operatorname{Cert}_{\text {ratee }}$.

\subsection{Reputation value updating protocol}

After Cert $_{\text {rater }}$ and Cert $_{\text {ratee }}$ are verified, reputation value of $P_{M}$ should be updated. But since the reputation account of $P_{M}$ is bound to the real identity $U_{M}$ of $P_{M}$. If 
$P_{M}$ updates reputation value directly, TTP will know $P_{M}$ is the temporary certificate of $U_{M}$, and reveal the link between user's temporary certificate and real identity. Assume that $\mathrm{U}$ colludes with TTP. $\mathrm{U}$ will know $P_{M}$ is the pseudonym of $M$, which poses grave threat to M's privacy. In order to solve this problem, blind signature is introduced when updating reputation value [28]. Two steps are adopted:

1) $P_{M}$ chooses random information $\mathrm{D}$, and sends it to TTP. TTP gives blind signature on D. $P_{M}$ acquires blind permission $\sigma . P_{M}$ deposits blind permission $\sigma$ in its own database.

$\mathrm{M}$ sends the blind permission $(D, \sigma)$ to TTP with its real identity $U_{M}$. TTP verifies whether the blind permission was used. If not, TTP updates the reputation value of $\mathrm{M}$ in reputation account.

\subsection{Attacker identifying \\ 6.7.1 Identifying the attacker}

If the certificate identification $\mathrm{S}$ in Cert $_{\text {rater }}$ equals certificate identification $\mathrm{V}$ in Cert $_{\text {ratee }}$, it means two temporary certificates of the same user are making mutual evaluations. Since in $S=F_{g, s}{ }^{D Y}\left(R_{1}\right)=g_{2}{ }^{1 / s+R_{1}+1}$ and $V=F_{g, s_{1}}{ }^{D Y}\left(R_{1}\right)=g_{2}{ }^{1 / s_{1}+R_{1}+1}, R_{1}$ is the same, and the secret of each node $\mathrm{s}$ is unique, then s must be the same with $s_{1}$. TTP can calculate the user's public key through two double evaluation equations $T=p k_{u} F_{g, s}{ }^{D Y}\left(R_{2}\right)^{r}=g_{2} s k_{u}+r /\left(R_{2}+s+1\right) \quad$ and $W=p k_{u} F_{g, s_{1}}{ }^{D Y}\left(R_{2}\right)^{t}=g_{2}{ }^{s k_{u}+t /\left(s_{1}+R_{2}+1\right)} \quad, \quad$ and generate violation proof $\Pi_{G}=\left(\pi_{1}, \pi_{2}\right)$. The calculation of the user's public key is as follows:

$p k=\left(\frac{T^{t}}{W^{r}}\right)^{(t-r)^{-1}}$

If $\mathrm{S}$ is the same with any $\mathrm{S}$ in Cert $_{\text {rater }}$ saved by TTP, it shows the frequency of mutual evaluations made between two users exceeds a limit. For Rater, $\left(r_{1}, T_{1}\right) \in \pi_{1}$, $\left(r_{2}, T_{2}\right) \in \pi_{2}$ have the same identification $\mathrm{S}$. Through two double evaluation

$T_{1}=p k_{u} F_{g, s}{ }^{D Y}\left(R_{2}\right)^{r_{1}}=g_{2}{ }^{s k_{u}+r_{1} /\left(R_{2}+s+1\right)}$

equations

$T_{2}=p k_{u} F_{g, s}{ }^{D Y}\left(R_{2}\right)^{r_{2}}=g_{2} s k_{u}+r_{2} /\left(R_{2}+s+1\right)$

and

calculates the identity of Rater and generates the violation proof $\Pi_{G}=\left(\pi_{1}, \pi_{2}\right)$. The calculation of the public key of Rater is as follows:

$p k_{\text {rater }}=\left(\frac{T_{2}^{r_{1}}}{T_{1}^{r_{2}}}\right)^{\left(r_{1}-r_{2}\right)^{-1}}$

For Ratee, $\left(t_{1}, W_{1}\right) \in \psi_{1},\left(t_{2}, W_{2}\right) \in \psi_{2}$ have the same identification $\mathrm{V}$. Through two double evaluation equations, $W_{1}=p k_{u} F_{g, s_{1}}{ }^{D Y}\left(R_{2}\right)^{t_{1}}=g_{2}{ }^{s k_{u}+t_{1} /\left(s_{1}+R_{2}+1\right)} \quad$ and
$W_{2}=p k_{u} F_{g, S_{1}}{ }^{D Y}\left(R_{2}\right)^{t_{2}}=g_{2}{ }^{s k_{u}+t_{2} /\left(s_{1}+R_{2}+1\right)}$, TTP calculates the identity of Ratee and generates the violation proof $\Pi_{G}=\left(\varphi_{1}, \varphi_{2}\right)$, The calculation of the public key of Ratee is as follows:

$p k_{\text {ratee }}=\left(\frac{W_{2}^{t_{1}}}{W_{1}^{t_{2}}}\right)^{\left(t_{1}-t_{2}\right)^{-1}}$

\subsubsection{Verifying violation proof}

Violation proof $\Pi_{G}=\left(\pi_{1}, \pi_{2}\right)$, where $\pi_{i}=\left(r_{i}, T_{i}, \phi_{i}\right)$. Operate Identity(params, $S, \pi_{1}, \pi_{2}$ ) algorithm, compare the output with the output of public key $p k_{u}$. Use it as input and check if these two match. Subsequently, verify $\phi_{i}$ related to $\left(S_{i}, r_{i}, T_{i}\right)$. If both are verified as valid, then VerifyGuilt(params, $S, \Pi_{G}, p k_{u}$ ) accepts the violation proof, otherwise it refuses the violation proof. TTP records the violator in the set $\Psi$

\subsection{Tracing malicious nodes}

Complete anonymity does not mean that none of evaluations of deals can be traced. If that is the case, such a system will bring convenience to outlaws. This paper has the following considerations when tracing malicious nodes: a) if it is a valid evaluation, TTP cannot identify the user's real identity, or trace the user; $b$ ) if the violation of a node occurs less than $d$ times in a limited amount of time, the node will not be traced; c) if the violation of a node occurs more than d times in a limited amount of time, the system will trace the violator. In order to trace a violator, TTP needs to acquire the key s of the violator's certificate container. The process is as follows:

\subsubsection{The generation of a sub-key}

By using Feldman's non-interactive verifiable key sharing, assume $\mathrm{d}$ is the pre-set threshold value. If the public key $p k_{u}$ of the violator first appears in the set $\Psi$ of TTP, the node needs to select a $d-1$ key sharing polynomial $f(x)$. The calculation of polynomial coefficient is: $k_{i}=1 /(s+R+1+i)$, where $R=H\left(p k_{u}\right)$, s is the secret of evaluation container, $i_{i}=0, \ldots, d$; the polynomial generated is $f(x)=k_{0}+k_{1} x+\ldots K_{d} x^{d} \bmod q$, $C_{i}=g^{k_{i}} \bmod p$. Take $\left\{C_{0}, \ldots, C_{d}\right\}$ as the coefficient's commitment value of the polynomial $f(x)$. The sub-key $s_{i}=\left(p k_{u},\left\{C_{i}\right\}_{i=0 \ldots d}, x, f(x), d\right), x=V=H\left(r_{i} \| T_{i}\right) . \mathrm{d}$ is the threshold value.

\subsubsection{Verifying the sub-key}

When the node updates its reputation value, if $p k_{u}$ is in the violators set $\Psi$ in the time limit $t(n)$, the peer must submit valid $s_{i}$. The number of $s_{i}$ submitted depends on the times of the number of $p k_{u}$ in $\Psi$ this time. Otherwise, 
TTP refuses to update the reputation value. The process of verifying the sub-key:

a) TTP verifies the received sub-key $\left(p k_{u},\left\{C_{i}\right\}_{i=0 \ldots d}, x, f(x), d\right)$. Through calculating $g^{f(x)}=C_{0} * C_{1}^{x} * \ldots C_{d} x^{d} \bmod p$, TTP verifies whether $(x, f(x))$ is the node of the polynomial $f(x)$ based on $\left\{C_{i}\right\}$ commitment. If not, TTP refuses the request of updating the node's reputation value.

b) If a sub-key with the public key being $p k_{u}$ already exists, TTP needs to verify whether $\mathrm{x}$ was used, and whether $\left\{C_{0}, \ldots, C_{d}\right\}$ are the same。

When the certificate is verified as valid, TTP updates $\Psi$, and deletes $p k_{u}$ from $\Psi$.

\subsubsection{The leaking of automatic threshold}

In the time period $\mathrm{t}$, for the same evaluation public key $p k_{u}$, if TTP acquires d sub-keys $s_{i}$, TTP can acquire the key $\mathrm{s}$ of the certificate container by using the $\mathrm{d}$ subkeys $s_{i}$, and reconstructing polynomial $f(x)$. The detailed process:

a) TTP reconstructs the polynomial $f(x)$ by using the d sub-keys $s_{i}$;

Extract the constant term $f(0)=k_{0}=1 / s+R+1$ of the polynomial $f(x)$, where $R=H\left(p k_{u}\right)$. The key of the container s can be acquired.

\subsubsection{Tracing malicious nodes}

TTP uses $S=F_{g, s}{ }^{D Y}\left(R_{1}\right)=g_{2}{ }^{1 / s+R_{1}+1}$ to calculate the evaluation certificate with the node's public key being $p k_{i}$, and can trace all evaluation and transaction records of the node with the public key being $p k_{i}$.

\section{Protocol analysis}

7.1 Validity analysis

Theorem 1: A node cannot generate an invalid evaluation certificate through a certificate container.

Proof: Adversary A and the knowledge extractor that acts as TTP $K=K_{\text {Withdraw }_{m, L_{S}}}^{B B-K(A)}$ (knowing the private key of TTP) execute $\mathrm{f}$ Withdraw . Assume that the first step proof of knowledge (commitment $C^{\prime}$ ) of Withdraw protocol is interactive, and $K^{`}=K_{\text {Withdraw }_{m, L_{S}}}^{B B-K(A)}$ is the extractor of knowledge proof. For the certificate withdrawal protocol Withdraw, except when $\mathrm{K}$ executes the code of $\mathrm{K}^{\prime}$ in the first step to extract the value from $\left(s k_{u}, s\right), \mathrm{K}$ is considered a truthful TTP. After acquiring secret s, and $\mathrm{K}$ can the calculation $\alpha=\left(S_{1}, S_{2} \ldots . S_{m}, s k_{u}, s\right)$ be accomplished, where $S_{i}=F_{g, s}{ }^{D Y}\left(R_{i}\right) \quad\left(R_{i}\right.$ is the temporary information on Ratee's temporary certificate), $C^{\prime}=\operatorname{Ped} \operatorname{Com}\left(S K_{u}, s^{\prime} ; r\right)$. When the execution of Withdraw protocol ends, $\mathrm{K}$ outputs $\left(\right.$ state $\left._{K}, C^{\prime}, \alpha\right) \in L_{S}$, where $C^{\prime}$ is the commitment, and equals $\operatorname{Ped} \operatorname{Com}\left(s K_{u}, s\right)$ the sent from adversary $A$ in the Withdraw protocol. Language $L_{S}$ is the triple $\left(., C^{\prime}, \alpha\right)$, where the first element can be of any value, but the second must be consistent with $\alpha$. $A_{f}=\left(S_{j, \mathrm{i}} \mid \leq j \leq f, 1 \leq i \leq n\right)$ is the identification after $\mathrm{f}$ executes Withdraw.

If the knowledge proof in Withdraw protocol is noninteractive, except the black box $A$ accesses, $\mathrm{K}$ and $\mathrm{K}$ ' are both controlled by random oracle.

$A_{f}$ includes all certificate tags of all deal-makers. For $A$ to win the game, it must make sure that TTP will accept an evaluation certificate of a non-truthful proof with a relatively high probability.

Assume $A$ is sure that a truthful TTP receives invalid identification $\mathrm{S}$ in reputation evaluation verifying protocol, where $S \notin A_{f}$. Then $A$ must forge a wrong proof: a) $A$ must know TTP's signature on public B, C; b) $\mathrm{S}$ and $\mathrm{T}$ will be generated in the proof $\Gamma$. Under the assumption that CL signature is secure (based on strong RAS assumption and LRSW), a) occurs with the probability that $V_{1}(k)$ is negligible; under the discrete assumption, b) occurs with the probability that $V_{2}(k)$ is negligible. Then $A$ occurs with the probability of $V_{1}(k)+V_{2}(k)$. Thus, the total success probability of $A$ is negligible.

\subsection{Anonymity analysis}

Theorem 2: The identity anonymity of Ratee and Rater should be ensured in executing the protocols.

Proof: Adversary $A$ can act as a corrupted TTP and Ratee, generate and publicize the public key $p k_{T T P}$. Adversary $A$ can execute Withdraw protocol for any times with any node. Eventually, adversary $A$ will execute evaluation certificate generation protocol RaterCert with some real Raters (having certificate container $W_{j}$ ) or simulator S (without $W_{j}$ ).

Simulator $S=S^{I O-R O(A)}$ (Params, auxsim, $\left.H\left(P_{i d}\right)\right)$ gives the input: public parameter params, supplementary information auxsim and $H\left(P_{i d}\right)$ ( $P_{i d}$ is the temporary certificate of Ratee). Given the random oracle model of the simulator and the input/output controlling model that adversary $A$ accesses, simulator $\mathrm{S}$ executes evaluation certificate generation protocol RaterCert that adversary $A$ requests in the following way:

a) Simulator $\mathrm{S}$ receives a character string info $\in\{0,1\}^{*}$;

b) Simulator $\mathrm{S}$ collects information on exchange, like info, $p k_{T T P}$, time, etc., the fixed output of $\mathrm{S}$ as a random oracle is a random value $r \in Z_{q}^{*}$;

c) S randomly chooses the secret $\mathrm{s}$ and calculates $S=F_{g, s}{ }^{D Y}\left(R_{1}\right)$ and $T=p k_{u} F_{g, s}{ }^{D Y}\left(R_{2}\right)^{r}$; 
d) $\mathrm{S}$ sends a reputation evaluation certificate Cert $_{\text {rater }}=(S, \pi=(r, T, \phi))$ to $A . \phi$ is the simulated signature proof generated through the following steps:

- $B=\operatorname{Ped} \operatorname{Com}\left(s k_{u}\right), \quad C=\operatorname{Ped} \operatorname{Com}(s)$ and a simulated proof that proves the commitment values of $\mathrm{B}$ and $\mathrm{C}$, which is based on CL signature of TTP; (in this step, $\mathrm{S}$ switches to $\mathrm{CL}$ simulator in random oracle model).

- A real proof $S=g^{1 / s+R_{1}+1}$ and the proof of $T=g^{s k_{u}+r /\left(R_{2}+s+1\right)} \Gamma$.

All the difficult problems of simulator $\mathrm{S}$ are knowledge proof problems about processing CL signature through the simulator. When withdrawing the protocol, due to CL signature, $A$ does not know the key s of the certificate container and the user's private key $s k_{u}$. Hence, the s simulator $\mathrm{S}$ chooses and the $\mathrm{s}$ the real user chooses are not mutually distinguishable. The components of evaluation certificate are Cert $_{\text {rater }}=(S, \pi=(r, T, \phi))$, where $\mathrm{r}$ is selected by adversary or RO model (which are not mutually distinguishable). Based on the security of DY PRF, the identification $\mathrm{S}$ in the evaluation certificate and the double evaluation equation $\mathrm{T}$ are not distinguishable from the random elements in $G_{2}$. Since $F_{g, s}{ }^{D Y}\left(R_{2}\right)^{r}$ is calculated in cyclic groups of prime numbers, $\mathrm{T}$ looks random. Simulated $\phi$ consists of two real proofs and a simulated proof. The simulation proof is the only difference between the simulator S's proof $\phi$ and the real user's proof $\phi$. Camenish Lysyanskaya simulator is operated to generate simulated proof. Due to the security of Camenish Lysyanskaya signature based on strong RSA or LRSW, the probability that $A$ distinguishes Game R (from real user) and Game I (from simulator S) is negligible.

\subsection{Identifying the attacker}

Theorem 3: the malicious attacker will be identified.

Proof: Since $r$ is randomly chosen by truthful Ratee $\mathrm{M}$, the probability that $r_{1}$ equals $r_{2}$ is very low. With regard to each double evaluation equation $T_{i}=p k_{u} F_{g, s}{ }^{D Y}\left(R_{2}\right)^{r}$ that has the same $p k_{u}$ and $\mathrm{s}$, the direct use of the correct Identity $\left(\gamma, S, \pi_{1}, \pi_{2}\right)$ algorithm can successfully restore the violator's identity $p k_{u}=g^{s k_{u}}$.

Ratee M (or random oracle) chooses $r_{1}$ and $r_{2}$. This determines the only $T_{1}=p k_{u} F_{g, s}{ }^{D Y}\left(R_{2}\right)^{r_{1}}=g^{s k_{u}+r_{1} /(s+1)}$ $T_{2}=p k_{u} F_{g, s}{ }^{D Y}\left(R_{2}\right)^{r_{2}}=g^{s k_{u}+r_{2} /(s+1)}$, which appears as the only valid and secure double evaluation equation with the evaluation identification $S$ in the two reputation evaluation certificates. In order to generate different identifications in executing the evaluation certificate generation protocol RaterCert, $A$ must change the valid proof $\pi$ in the $4^{\text {th }}$ step of the certificate generation protocol (in "validity proof", it has already been proven that the probability is negligible).
Identity algorithm can output the public key $p k_{u}$ of the attacker, and the malicious Rater's proof $\Pi_{G}=\left(\pi_{1}, \pi_{2}\right)$, because VerifyGuilt accepts only truthful Identity output, and truthful public key $p k_{u}$ and $\Pi_{G}$ will be accepted by VerifyGuilt(params, $S, \Pi_{G}, p k_{u}$ ) with a relatively high probability.

\subsection{Non-frameability analysis}

Theorem 4: The forged evaluation certificate proof of other nodes can be identified.

Proof: Any valid proof $\pi_{i}$ should concern the knowledge proof of the user's private key (refer to evaluation certificate generation protocol). If the two reputation evaluation certificates $\left(S, \pi_{1}\right)$ and $\left(S, \pi_{2}\right)$ of the violation proof $\prod_{G}=\left(\pi_{1}, \pi_{2}\right)$ are accepted by a truthful TTP, it means there are two possibilities: 1) adversary $A$ must successfully forge the knowledge proof. In "validity proof", it has already been proven that the probability is negligible; 2) if $\left(S, \pi_{1}\right)$ and $\left(S, \pi_{2}\right)$ are accepted as different reputation evaluation certificates for they are registered as different users, VerifyGuilt algorithm will refuse $\Pi_{G}=\left(\pi_{1}, \pi_{2}\right)$, because Identity algorithm cannot restore the user's public key $p k_{u}$ through the double evaluation equation generated by different public keys $p k_{u}$ and secret $\mathrm{s}$.

\section{Comparison and contrast}

This paper adopts similar schemes to [31] and [32]. This chapter mainly compares this scheme with that of [31] and [32] in efficiency, anonymity and security.

\subsection{Efficiency comparison}

Table 1 Efficiency comparison

\begin{tabular}{|c|c|c|c|}
\hline Scheme & \multicolumn{2}{|c|}{$\begin{array}{c}\text { Calculation } \\
\text { (modular exponent) }\end{array}$} & $\begin{array}{l}\text { Complexity } \\
\text { (Messages) }\end{array}$ \\
\hline \multirow{2}{*}{ The scheme } & Rater & 11 & \multirow{2}{*}{$O(1)$} \\
\hline & TTP & 16 & \\
\hline \multirow{2}{*}{ [31] scheme } & Rater & 27 & \multirow{2}{*}{$O(1)$} \\
\hline & TTP & 16 & \\
\hline \multirow[b]{2}{*}{ [32] scheme } & Rater & more than 30 & \multirow[b]{2}{*}{$O(1)$} \\
\hline & $\begin{array}{c}\text { reputation } \\
\text { value recipient }\end{array}$ & more than 30 & \\
\hline
\end{tabular}

The calculation of this scheme, and those from [31] and [32] is mainly on multi-based modular exponent and single-based modular exponent. But through proper methods, calculations like $g_{1}{ }^{x_{1}} g_{2}{ }^{x_{2}}$ can be taken as 1,2 single modular exponent, and calculations like $g_{1}^{x_{1}} g_{2}^{x_{2}} g_{3}^{x_{3}}$ can be taken as 1,5 modular exponent.

Considering that the evaluation certificate container withdrawal protocol in this scheme, e-cash withdrawal protocol in [31] and user registration protocol in [32]can all be executed offline, the comparison on efficiency will mainly focus on the executing efficiency of these three 
schemes in terms of reputation evaluation and reputation verifying protocol.

In this scheme, Rater needs to execute modular exponent calculations for 11 times to generate reputation evaluation certificate. TTP needs to execute modular exponent calculations for 16 times to verify the validity of the evaluation certificate and the Ratee certificate; in [31], Rater needs to execute modular exponent calculations for 27 times to generate e-cash that acts as evaluation value. In the reputation evaluation verifying protocol, TTP needs to execute modular exponent calculations for 16 times to verify the validity of e-cash; in [32], reputation Rater needs to execute modular exponent calculations for more than 30 times to generate the signature of reputation value, and the reputation value recipient also needs to execute modular exponent calculations for more than 30 times to verify the signature of the reputation value. Compared with [31] and [32], in terms of the complication of calculation, this scheme has made improvements.

In terms of the complication of information, the three schemes are basically the same.

\subsection{Comparison of anonymity and security}

Both this scheme and [32] adopt zero-knowledge proof and blind signature technology to realize the anonymity of the Ratee. Though [31] adopts e-cash technology, this technology is based on zero-knowledge proof. Thus, the privacy technology used in these three schemes is similar; all three realized the anonymity of Ratee. But in terms of security, this scheme can resist and identify Sybil attack and ballot-stuffing attack. The work in [31] cannot resist or identify any malicious attack. And [32] can only resist Sybil attack.

Table 2 Anonymity and security

\begin{tabular}{|c|c|c|c|}
\hline Scheme & Key Security Mechanisms & Ratee's anonymity & Security \\
\hline This scheme & Zero-knowledge proof, blind signature & supportive & $\begin{array}{c}\text { Resisting and identifying Sybil and ballot- } \\
\text { stuffing attack }\end{array}$ \\
\hline$[31]$ scheme & e-cash & supportive & Not being able to identify the attacker \\
\hline$[32]$ scheme & Zero-knowledge proof, blind signature & supportive & Resisting Sybil attack \\
\hline
\end{tabular}

\section{Conclusion}

This paper addresses such problems as the conflict between anonymity and reputation and the uncontrollability of reputation evaluation that are prominent in reputation system where nodes' identity is absolutely anonymous. It introduces conditional anonymity into reputation system to anonymously monitor the reputations evaluations made by users. Only when the anonymity condition is met can the reputation value be gained. Violators will be exposed and punished. Nodes that exceed the violation frequency in a certain time limit will be traced. Reputation evaluations made between users will be controlled. Based on blind signature, when a user updates the reputation value, its privacy can be protected, and its temporary certificates unlinked. It can also reconcile the conflict between anonymity and reputation.

\section{Reference}

[1] Jøsang, A.; Ismail, R.; Boyd, C. A survey of trust and reputation systems for online service provision. // Decision Support Systems. 43, 2(2007), pp. 618-644. DOI: 10.1016/j.dss.2005.05.019

[2] Seigneur, J.; Jensen, M. Trading Privacy for Trust. // The International Conference on Trust Management Proceedings of iTrust'04, Oxford, UK. vol. 2995, (2004), pp. 93-107. DOI: 10.1007/978-3-540-24747-0_8

[3] Gupta, M.; Judge, P.; Ammar, M. A reputation system for peer-to-peer networks. // NOSSDAV '03 Proceedings of the $13^{\text {th }}$ international workshop on Network and operating systems support for digital audio and video, New York, 2003, pp. 144-152.

[4] Aberer, K.; Despotovic, Z. Managing Trust in a Peer-2-Peer Information System. // In Proc. of the $10^{\text {th }}$ International Conference on Information and Knowledge Management (CIKM01) / New York, 2001, pp. 310-317. DOl: $10.1145 / 502585.502638$
[5] Steinbrecher, S. Enhancing multilateral security in and by reputation systems. // In FIDIS/IFIP Internet Security and Privacy Summer School. 2008, pp. 135-150.

[6] Pingel, F.; Steinbrecher, S. Multilateral secure crosscommunity reputation systems for internet communities. // Proceedings of the Fifth International Conference on Trust and Privacy in Digital Business (TrustBus2008) / Italy. Vol. 5185, (2008), pp. 69-78. DOI: 10.1007/978-3-540-85735-8_8

[7] Steinbrecher, S. Design options for privacy-respecting reputation systems within centralised internet communities. // Security and Privacy in Dynamic Environments, Karlstad, Sweden. Vol. 201, (2006), pp. 123-134. DOl: 10.1007/0-38733406-8_11

[8] Damiani, E.; di Vimercati, S. D. C.; Paraboschi, S. et al. Managing and sharing servents' reputations in $\mathrm{p} 2 \mathrm{p}$ systems. // IEEE Transaction on Knowledge and Data Engineering. 15, 4(2003), pp. 840-854. DOI: 10.1109/TKDE.2003.1209003

[9] Kamvar, S. D.; Schlosser, M. T.; Garcia-Molina, H. The eigentrust algorithm for reputation management in $\mathrm{P} 2 \mathrm{P}$ networks. // Proc. of the $12^{\text {th }}$ Int'l World Wide Web Conf. / Budapest. 2003, pp. 640-651.

[10] Kinateder, M.; Terdic, R.; Rothermel, K. Trusting pseudonyms-anonymous communication in Peer-to-Peer reputation systems. // International Journal for Infonomics. (2005), pp. 1-13.

[11] Ismail, R.; Boyd, C.; Jøsang, A. et al. A Security Architecture for Reputation Systems. // Proceedings of the $4^{\text {th }}$ International Conference of E-Commerce and Web Technologies, Prague, Czech Republic. vol. 2738, (2003), pp. 176-185. DOI: 10.1007/978-3-540-45229-4_18

[12] Ismail, R.; Boyd， C.; Jøsang, A.; Russel， S. Private reputation shemes for P2P systems. // In WOSIS, 2004/2004, pp. 196-206.

[13] Androulaki, E.; Choi, S.; Bellovin, S. et al. Reputation Systems for Anonymous Networks. // Proceedings of the $8^{\text {th }}$ International Symposium on Privacy Enhancing Technologies, Leuven, Belgium, vol. 5134, (2008), pp. 201-218. DOI: 10.1007/978-3-540-70630-4_13

[14] Santos, Q.; Hasan, O.; Brunie, L. A Distributed PrivacyPreserving Reputation Protocol Resistant to Reflection Attacks. // Rapport de recherche RR-LIRIS-2014-004, 2014. 
[15] Petrlic, R.; Lutters, S.; Sorge, C. Privacy-Preserving Reputation Management. // In: Proceedings of the $29^{\text {th }}$ Symposium on Applied Computing, Gyeongju, Korea. 2014, pp. 1712-1718. DOI: $10.1145 / 2554850.2554881$

[16] Vasirani, M.; Wijaya, T. K.; Liassas, G. et al. Privacy Enhanced Demand Response with Reputation-based Incentive Distribution. // In: International Workshop on Demand Response, Cambridge, UK. 2014, pp. 1-6.

[17] Voss, M. Privacy preserving online reputation systems. // In International Information Security Workshops, Toulouse, France. vol. 148, (2004), pp. 245-260.

[18] Chaum, D.; Fiat, A.; Naor, M. Untraceable electronic cash. // In CRYPTO'90, Santa Barbara. California, USA. vol. 403, (1990), pp. 319-327.

[19] Camenisch, J.; Hohenberger, S.; Lysyanskaya, A. Compact E-Cash. // In EUROCRYPT '05. vol. 3494, (2005), pp. 302321.

[20] Jarecki, S.; Lincoln, P.; Shmatikov, V. Negotiated Privacy Software Security: Theories and Systems: Mext-NSF-JSPS International Symposium. // In: ISSS 2002, Tokyo, Japan. 2002, pp. 8-10.

[21] Jarecki, S.; Shmatikov, V. Handcuffing big brother: an abuse-resilient transaction escrow scheme. // In EUROCRYPT, Interlaken, Switzerland. vol. 3027, (2004), pp. 590-608.

[22] Nguyen, L.; Safavi-Naini, R. Dynamic k-times anonymous authentication. // In ACNS. vol. 3531, (2005), pp. 318-333.

[23] Camenisch, J.; Hohenberger, S.; Kohlweiss, M. et al. How to win the clonewars: efficient periodic n-times anonymous authentication. // In: $13^{\text {th }}$ ACM Conference on Computer and Communications Security, Alexandria, VA, USA, 2006, pp. 201-210. DOI: 10.1145/1180405.1180431

[24] Fujisaki, E.; Okamoto, T. Statistical zero knowledge protocols to prove modular polynomial relations. //I n Advances in Cryptology CRYPTO '97, Santa Barbara, California. vol. 1294, (1997), pp. 16-30.

[25] Camenisch, J.; Lysyanskaya, A. A signature scheme with efficient protocols. // In: Security in Communication Networks '02, Amalfi, Italy. vol. 2576, (2002), pp. 268-289.

[26] Dodis, Y.; Yampolskiy, A. A Verifiable Random Function with Short Proofs Keys. // In Public Key Cryptography. Vol. 3386 of LNCS, (2005), pp. 416-431.

[27] Pedersen, T. P. Non-interactive and information-theoretic secure verifiable secret sharing. // In CRYPTO '92. vol. 576, (1992), pp. 129-140.

[28] Juels, A.; Luby, M.; Ostrovsky, R. Security of blind digital signatures. // In Advances in Cryptology CRYPTO '97, Santa Barbara, California. vol. 1294, (1997), pp. 150-164.

[29] Fiat, A.; Shamir, A. How to prove yourself: Practical solutions to identification and signature problems. // In Advances in Cryptology CRYPTO '86, Santa Barbara, California, USA. vol. 263, (1986), pp. 186-194.

[30] Camenisch, J.; Stadler, M. Efficient group signature schemes for large groups. // In CRYPTO '97, Santa Barbara, California, USA. vol. 1296, (1997), pp. 410-424.

[31] Androulaki, E.; Choi, S.; Bellovin, S. et al. Reputation Systems for Anonymous Networks. // Proceedings of the $8^{\text {th }}$ International Symposium on Privacy Enhancing Technologies, Leuven, Belgium. vol. 5134, (2008), pp. 201-218. DOI: 10.1007/978-3-540-70630-4_13

[32] Bethencourt, J.; Shi, E.; Song, D. Signatures of Reputation: Towards Trust without Identity. // In: Sion, R. (ed.) FC 2010 / Heidelberg. 6052, (2010), pp. 400-407.

\section{Authors' addresses}

Keli Zhang, PhD Candidate

Information Security Center,

Beijing University of Posts and Telecommunications, No 10, Xitucheng Road, Haidian District,

100876 Beijing, P. R. China

E-mail: kelicybergirl@163.com

Yi Xian Yang, Professor

Information Security Center,

Beijing University of Posts and Telecommunications, No 10, Xitucheng Road, Haidian District, 100876 Beijing, P. R. China

E-mail: yangyixian@bupt.edu.cn 